\title{
Inserción laboral y diversidad funcional: un estudio compostelano con perspectiva de género
} \section{gender perspective \\ Raquel Mariño Fernández y Gemma González Río Universidade de Santiago de Compostela, España}

Labor insertion and functional diversity: a Compostela study with a

$\left(\begin{array}{l}\text { Fecha de recepción: } 17 \text { de octubre de } 2020 \\ \text { Fecha de aprobación: } 24 \text { de noviembre de } 2020\end{array}\right)$

DOI: http://dx.doi.org/10.15304/ricd.3.13.7264

\section{NOTAS BIOGRÁFICAS}

Raquel Mariño Fernández es licenciada en Psicopedagogía y doctora en Ciencias de la Educación por la Universidad de Santiago de Compostela. Su trayectoria formativa y profesional están relacionada con cuestiones vinculadas a la didáctica, la orientación, el género y diversidad y la Formación Profesional y para el Trabajo. En la actualidad es profesora en el Departamento de Pedagogía y Didáctica- Área de Didáctica y Organización Escolar- Facultad de Ciencias de la Educación de la Universidad de Santiago de Compostela.

Contacto: raquel.marino@usc.es

Gemma González Río es graduada en Educación Social por la Universidad de Santiago de Compostela. Realiza voluntariado vinculado con el ámbito de la salud mental en la entidad AGADEA -Alzhéimer. estimulación terapéutica. En estos momentos se encuentra formándose como alumna en el Máster Profesional (Instituto SIAC): Intervención con Menores en Riesgo Social.

Contacto:gemma.gonzalez.rio@rai.usc.es

\section{Resumen}

El empleo se considera un eje central de la inclusión y su ausencia conlleva una serie de carencias sociales, educativas, de participación e igualdad, que desembocan en una vulnerabilidad social. En el caso de las mujeres, su ausencia multiplica la incidencia de otras variables que dificultan su plena inclusión social.

La Agenda 2030 (ONU, 2015), establece 17 Objetivos de Desarrollo Sostenible (ODS) que, de forma transversal, incluyen en su objetivo cincola perspectiva de género. En pleno transcurso a favor del desarrollo sostenible, nos planteamos estudiar el cómo se están llevando a cabo acciones locales en la ciudad de Santiago de Compostela, que contemplen la inclusión laboral de mujeres con diversidad funcional.

Para ello, seguimos un enfoque metodológico de doble vertiente: se realiza un análisis profundo de normativa, programas y recursos web de instituciones destacadas del ámbi to compostelano; y se encuesta a informantes clave para conocer los aspectos que favorecen la inserción laboral de este 
colectivo. Las entidades participantes son Down Compostela, FADEMGA Plena Inclusión Galicia, COGAMIy ACADAR.

\section{Abstract}

Employment is considered a central axis of inclusion and its absence entails a series of social, educational, participation and equality deficiencies, which lead to social vulnerability. In the case of women, their absence multiplies the incidence of other variables that hinder their full social inclusion.

The 2030 Agenda (ONU, 2015), establishes 17 Sustainable Development Objectives (SDO) that, in a transversal way, include the gender perspective in their objective five. In the middle of the process in favor of sustainable development, we plan to study how local actions are being carried out in the city of Santiago de Compostela, which contemplate the labor inclusion of women with functional diversity.

To do this, we follow a two-pronged methodological approach: a deep analysis of regulations, programs and web resources of leading institutions in the Compostela area is carried out; and key informants are surveyed to find out the aspects that favor the employment of this group. The participating entities are Down Compostela, FADEMGA Plena Inclusión Galicia, COGAMI and ACADAR.

Palabras clave

Inserción laboral, diversidad funcional, género, igualdad, formación, intervención socioeducativa.

Keywords

Labor insertion, functional diversity, gender, equality, education, socioeducational intervention.

\section{Sumario}

1. Introducción

2. Metodología

\subsection{Instrumentos}

\subsection{Muestra}

3. Discusión de los resultados obtenidos

4. Conclusiones finales: un decálogo de buenas prácticas

\section{Summary}

1. Introduction

2. Methodology

2.1. Instruments

2.2. Sample

3. Discussión of the obtained results

4. Final conclusions: a decalogue of good practices 


\section{INTRODUCCIÓN}

El término "diversidad funcional" es un concepto relativamente reciente en la sociedad que, actualmente, coexiste con otros conceptos afines entre los cuales el más común sigue siendo el de "discapacidad". A lo largo de la historia de la humanidad, la concepción en torno a esta temática pasó por diferentes modelos, estados y conceptualizaciones que no acaban de desligarse del aspecto connotativo de "personas con una enfermedad que se debe curar". Podemos decir que los estereotipos y estigmas asociados a este colectivo continúan muy arraigados en la forma de ver y concebir a estas personas, lo que juega un papel determinantea la hora de lograr mudarsu denominación conceptual de inferioridad y/o la falta de respeto a su dignidad.

Partiendo de la línea de estos estereotipos y estigmas asociados a dicho colectivo, se hace imprescindible cambiar ya la negativa denominación conceptual que se utiliza para referirse a las personas con diversidad funcional. Tradicionalmente, se han utilizado adjetivos y apelativos que como personas las degradaban y las situaban en una posición de inferioridad frente a las demás. Actualmente, esas denominaciones, en su mayoría peyorativas, cayeron en desuso y predomina el uso de términos más amigables como el de "diversidad funcional".

Hoy en día se sigue trabajando en esta dirección y es posible percibir los avances en el plano legislativo, donde surgen nuevas medidas que buscan erradicar las discriminaciones que aún prevalecen hacia este colectivo, como también en el plano social, donde los diversos profesionales siguen comprometidos con la tarea de visibilizar al colectivo para comprender la magnitud del problema subyacente (Romañach y Palacios, 2008). Todos los progresos y triunfos alcanzados permiten, en su conjunto, articular un nuevo escenario desde el cuál comenzar a construir una nueva mentalidad y acciones cara una inclusión digna de este colectivo. Este último aspecto resulta excepcionalmente relevante para las ciencias sociales, pues provoca que el tratamiento de la diversidad funcional, bajo un enfoque de género, sea un ámbito de estudio en alza que explore más realidades que las abarcadas hasta el momento (Soler, Teixeira y Jaime, 2008).

También nos resulta un escenario de estudio excepcional el que se nos brinda para estos trabajos desde la Agenda 2030, a probada en el año 2015 yen el seno de la Naciones Unidas. En ella se establecen 17 Objetivos de Desarrollo
Sostenible (ODS) que, de forma transversal, incluyen en su objetivo número cinco la inclusión de la perspectiva de género. Por consiguiente, la oportunidad para visibilizar la realidad sociolaboral que viven las mujeres con diversidad funcional, también encuentra surazón de ser en los grandes principios sobre los que se asienta la Agenda 2030, los cuales se delimitan en: la universalidad, la integralidad y el "no dejar a nadie atrás". Así, para este trabajo resultan de crucial interés dichos principios, pero también los ODS 4, 5, 8 y 10 que se corresponden con el logro de una educación de calidad; el logro de la igualdad de género; el logro del trabajo decente y crecimiento económico y, finalmente, el logro de la reducción de las desigualdades.

Si a mayores tenemos presente lo que se recoge en el Informe sobre Desarrollo Humano del Programa de las Naciones Unidas para el Desarrollo (PNUD,2016), nos encontramos con que se hace indispensable cuidar de aquellas personas que se han quedado atrás, diseñando estrategias y políticas que articulen medidas de actúación en consonancia con cuatro ejes de desarrollo: a) redactar políticas universales que fomenten el crecimiento sosteniblee inclusivo; b) incentivar medidas locales dirigidas a los grupos con necesidades especiales; c) proteger al ser humano frente a las adversidades; d) ayudar a empoderar a los colectivos excluidos. Las mujeres con diversidad funcional se ven privadas, en gran parte, de todas estas medidas porque continúan discriminadas, estigmatizadas y excluidas en gran parte de las esferas sociales y laborales. Todo esto aún nos imposibilita hablar de triunfos totales en materia de equidady desarrollo humano a nivel local, autonómicoy estatal.

Por suerte, nos encontramos en un período temporal en donde, aunque faltan menos de diez años para el cumplimiento de los ODS, es una década importante de acción y resultados que siguen apostando por el desarrollo sostenible. Por ello, nos hemos planteado aprovechar esta circunstancia y estudiar las acciones locales de instituciones clave, que contemplen la inclusión laboral de mujerescon diversidad funcional, en el entorno de la ciudad de Santiago de Compostela. Para comprender la necesidad de llevar a cabo dicho estudio, se debe conocer la relación existente entre la diversidad funcional, las mujeres y las acciones locales de inserción laboral que se están llevando a cabo desde un marco de desarrollo sostenible.

En el ámbito de la educación social, la inserción laboral de las personas con diversidad funcional constituye una problemática severa que 
repercute en el desarrollo de una vida plena para ellas. Es decir, el empleo se considera un eje central de la inclusión y su usencia conlleva una serie de carencias sociales, educativas, de participación e igualdad, que desembocan en una vulnerabilidad social. Éste es un elemento especialmente sensible en el caso de las mujeres, ya que multiplica la incidencia de otras variables que dificultan una inclusión plena en la sociedad. A los prejuicios derivados de la diversidad funcional, se le unen el arraigo sociocultural de determinados estereotipos sobre la imagen y el papel que deben desempeñar las mujeresen las esferas productivas y/o domésticas, o sobre su supuesto menor rendimiento y/o falta de capacidad para formar parte del tejido empresarial, su elevada dependencia o ya, para rizar más el rizo, su falta de destrezas para el logro de una emancipación plena, entre otras barreras y discriminaciones. Todos estos aspectos influyen negativamente en la construcción social de este colectivo $\mathrm{y}$, desde un punto de vista individual, en la construcción de su propio autoconcepto yautoestima (Portillo, Shum, Conde y Lobato, 2006).

Como consecuencia, se derivan carencias a distintos niveles en lo formativo, legislativo, laboral y social, que contribuyen a dificultar las oportunidades reales de inserción laboral de estas mujeres. Como afirman Carrizosa y Sesé (2014), nos encontramos en un sistema social que valora a la ciudadanía por sus capacidades de trabajo y consumo, dejando apartadas a aquellas personas que, por diversos factores, no pueden participar en igualdad de oportunidades. En el caso de este colectivo, esta realidad se ve agravada por la sobreprotección que les rodea, lo que acentúa la tendencia a considerarlas dependientes, poco capaces y más vulnerables (Serra, 2014). Las acciones discriminatorias a las que se enfrentan en su proceso de búsqueda de empleo, solo representan uno de los ejes de exclusión que las rodean pues, tal como argumenta Cedrero (2017, p. 247), "los factores discriminatorios son una expresión de la interseccionalidad de género que sufre la mujer con diversidad funcional en el ámbito laboral, y se manifiestan en bajas tasas de ocupación, menor estabilidad en el empleo, discriminación retributiva, maltratos psicológicos y agresiones sexuales".

Al consultar los datos del Instituto Nacional de Estadística (2018), nos encontramos con qué en España, a partir de los 16 años, un 18,6\% de la población presenta algún tipo de diversidad funcional. Si los datos los filtramos por sexos, nos encontramos con que un $16,4 \%$ de los hombres presentan diversidad funcional frente al $20,8 \%$ de las mujeres. En el caso de los datos referidos a la tasa de actividad, observamos que este dato es inferior en el caso de las mujeres $(41,6 \%)$ frente al 58,4\% que representa esta tasa para el colectivo masculino. En la misma tesitura observamos que la tasa de paro es cuatro puntos superiores en el caso femenino (17,1\% de mujeres), frente al 13,8\% que registran los hombres. Esta información se complementa con lo recogido en el Informe Olivenza (2019), donde se indica que, en el año 2018 , un $25 \%$ de las personas ya presentaban algún tipo de limitación en las actividades cotidianas debido a problemas de salud. En dicho informe, el colectivo femenino también presentaba una mayor tendencia en las limitaciones de actividad $(27,3 \%$ de mujeres frente a un $22,6 \%$ de hombres). Estas dificultades, añadidas a las barreras que se derivan de la doble discriminación condicionan que, a pesar de ser las mujeres el grupo mayoritario dentro de la diversidad funcional, presenten unos valores de desempleo superiores, mayor tendencia a la inestabilidad y mayores tasas de precariedad laboral.

Por todas estas razones, el objetivo principal que ha guiado todo este proceso fue conocer y visibilizar la situación laboral real en la que se encuentran las mujeres con diversidad funcional de esta comarca para, a partir de los resultados, elaborar un decálogo de directrices y buenas prácticas que permitan mejorar e incentivar las acciones de desarrollo local, para la inserción laboral de las mujeres con diversidad funcional en la ciudad de Santiago de Compostela. Para poder lograr dicho objetivo se han analizado desde las actuaciones reguladas en el marco legislativo, hasta las líneas de intervención, programas y acciones de inserción laboral llevadas a cabo en instituciones reales con este colectivo. Todo este análisis documental, institucional y profesional nos ha permitido conocer la viabilidad y realidad desde la que trabajan los y las profesionales de las instituciones seleccionadas, así como los logros alcanzados hasta este momento.

\section{METOdologíA}

Para llevar a cabo este estudio, de corte empírico y cualitativo, se utilizó una metodología de doble vertiente, que nos permitió: por un lado, apoyar la investigación en una revisión bibliográfica (teórica y normativa) profunda para, a partir de la misma, abordar la realidad de las entidades de una forma más verídica; y, por el otro lado, enfocar la investigación hacia una parte más cualitativa dónde, a partir de entrevistas a infor- 
mantes clave, se lograse obtener datos de primera mano y basados en las propias vivencias, percepciones y experiencias de los y las profesionales que allí trabajan y que, de otra forma, no hubiera sido factible conocer.

\subsection{INSTRUMENTOS}

En el desarrollo de este estudio era importante conocer y dominar tanto la normativa legal vigente, como lo que ofrecen en sí las instituciones y sus equipos de profesionales.

Ello supuso diseñar dos instrumentos principales que nos permitieran recoger información relevante sobre las entidades en sí y, a su vez, sobre sus propias actuaciones, recursos y resultados. Así, se decide elaborar una guía para el análisis de las páginas web y recursos de las entidades y una encuesta semiestructurada para los informantes clave. Con respecto a la guía podemos decir que, a partir del modelo empleado en el proyecto de investigación "Escuel@ digit@|1", se confecciona una guía estructurada en seis bloques centrales referidos a: datos de la entidad y autoría del recurso, datos e información específica sobre el tipo de recurso, estructura, contenidos que ofrece y usos que se hacen con los mismos. En cuanto al guion de la entrevista, podemos decir que se estructura en veinte preguntas pertenecientes a cuatro bloques: el primero, introduce cuestiones como la formación y el puesto laboral que ocupa en la institución; el segundo, aborda la labor desarrollada por los informantes clave en los puestos laborales que ocupan dentro de las instituciones; el tercero indaga sobre las relaciones, actitudes e implicación que muestran las familias anteel proceso de inserción laboral de estas mujeres, el trabajo de ruptura de estereotipos y la mejora de la autoestima; el cuarto bloque cierra la entrevista con cuestiones que buscan conocer las dificultades y adversidades que encuentran y afrontan en su labor diaria, así como las ayudas externas a las que recuren y/o las mejoras que consideran necesarias acometer en sus líneas de intervención.

Ambos instrumentos persiguieron ampliar, contrastar y complementar información y datos que nos permitiesen un conocimiento más profundo de las acciones y líneas de intervención de las cuatro entidades seleccionadas: Down Compostela, FADEMGA-Plena Inclusión Galicia, COGAMI y ACADAR. Los resultados obtenidos fueron puestos en valor y relacionados con los objetivos iniciales, permitiéndonos elaborar unas conclusiones finales, así como proponer una serie de buenas prácticas para mejorar la inserción laboral del colectivo femenino con diversidad funcional.

\subsection{MUESTRA}

En cuanto a las entrevistas realizadas, se seleccionan, en primer lugar, aquellas entidades que más antigüedad y relevancia presentan por su trayectoria en materia de inserción laboral de mujeres con diversidad funcional. A continuación, se contacta con ellas y se busca el perfil de profesionales que puedan ser considerados como informantes clave. Tras varias consultas, cada entidad nos facilita el contacto con el profesional que, bajo su criterio, trayectoria y experiencias, puede ser la persona más idónea como informante clave para abordar dicha problemática. De este modo, se contacta con cuatro profesionales, pertenecientes a las cuatro instituciones de referencia, a las que se les envía previamente el guion de la entrevista y se establece una cita para realizarla. Con respecto a su perfil podemos decir que todas ellas son mujeres y ocupan puestos de coordinadora en áreas de empleo y formación. Las titulaciones base que poseen son Grado en Psicología, Económicas, Trabajo Social, Psicopedagogía y Magisterio.

Con respecto al perfil de sus demandantes de empleo nos dicen quetodas las mujeresa las que atienden ya eran usuarias de sus servicios. En términos globales nos indican que atienden a un $40 \%$ de mujeres frente a un $60 \%$ de hombres (excepto en el caso concreto de ACADARque sólo trabaja con el colectivo femenino). En cuantoa su procedencia, nos indican que pertenecen al área compostelana, sobre todo al núcleo urbano, pues en los alrededores más rurales, dada su dispersión geográfica, el acceso a las entidades físicas y a los núcleos laborales es más difícil al depender de un medio de transporte o de una persona que las aproxime al lugar. En cuanto al nivel formativo del que parten estas mujeres demandantes, las informantes nos comentan que casi todas ellas cuentan con estudios primarios.

\section{DISCUSIÓN DE LOS RESULTADOS OBTENIDOS}

En cuanto a los resultados obtenidos, podemos decir que cuando se ha revisado el marco legal que regula la inserción laboral de las mujeres con diversidad funcional, así como el tipo de 
actuaciones que se contemplan en el mismo para una inserción real, hemos detectado que la mayoría de las medidas recogidas en esta normativa, tanto a nivel estatal como autonómico, provienen de lo que se conoce como iniciativas sociales. Estas iniciativas responden a diversos programas de intervención social, territoriales y de desarrollo local, que buscan promover la mayor participación social y económica de las mujeres. Para ello tienen presente su propia diversidad así como las características que definen el entono sociocultural en el que habitan, sobre el cual pretenden llevar a cabo los procesos de integración, inclu- sión e inserción; Aunque son programas que llevan más de una década legislados con fondos económico considerables, su análisis en profundidad nos puso en relevancia la falta de medidas legislativas concretas que, desde los órganos gubernamentales, amparen con mayor eficacia y calidad la situación laboral de las mujeres con diversidad funcional. Si bien es cierto que, en determinadas leyes, se hace alusión en alguno de sus artículos a cuestiones puntuales sobre su situación laboral, son relativamente pocos los ejemplos existentes sobre medidas centradas en esta área con respecto al colectivo en cuestión.

Tabla 1

Normativa consultada.

\begin{tabular}{llll}
\hline BOLETín OfICIAL DO ESTADO (BOE) & $\begin{array}{l}\text { DIARIO OFICIAL DE GALICIA } \\
\text { (D OG) }\end{array}$ & $\begin{array}{l}\text { XUNTA DE } \\
\text { GALICIA }\end{array}$ & PÁXINAS WEB \\
\hline Ley 51/2003, de 2 de & Lei 12/2016, do 22 de & $\begin{array}{l}\text { III Plan de Igualdade } \\
\text { de Oportunidades das }\end{array}$ & $\begin{array}{l}\text { Estrategia Española } \\
\text { sobre Discapacidad }\end{array}$ \\
diciembre, de lgualdad de & $\begin{array}{l}\text { xullo, pola que se } \\
\text { modifica a Lei 11/2007, }\end{array}$ & Mulleres Galegas \\
Discriminación y & de 17 de xullo, galega & 1998-2001. & $2012-2020$. \\
Accesibilidad Universal de & para a prevención e o & &
\end{tabular}

las Personas con

Discapacidad (LIONDAU).

Ley Orgánica 3/2007, de 22

de marzo, para la igualdad

efectiva de mujeres y

hombres.

Constitución Española de 27 de diciembre de 1978. tratamento integral da

violencia de xénero.

Lei $2 / 2007$, de 28 de marzo, do Traballo en Igualdade das mulleres de Galicia.

Lei $7 / 2004$, de 16 de xullo, Galega para a Igualdade de Homes e Mulleres.
IV Plan de Igualdade de Oportunidades das mulleres galegas

(2002-2005)

V Plan do Goberno Galego para a Igualdade entre Mulleres e Homes 2007-2010.

VII Plan Estratégico de Galicia para la Igualdad de Oportunidades entre Mujeres y Hombres 2017-2020.

Elaboración propia.

I Plan Integral de Acción de Mujeres con Discapacidad 20052008. Madrid: CERMI.
II Plan de Acción para las Personas con Discapacidad 2003-2007.

II Plan de Acción para personas con síndrome de Down en España 2009-

2013.

Plan Integral de Acción de mujeres con discapacidad 2013-2016. Madrid: CERMI 
A modo de resumen, como principales áreas que se contemplan en dichas normativas legales hemos identificado la igualdad, la autonomía, la visibilización y sensibilización en torno a la situación del colectivo, y la eliminación de barreras que dificultan su acceso al mundo laboral, entre otras. En cuanto a las líneas de intervención, éstas se dirigen fundamentalmente hacia el logro de: a) una mejora formativa, con el objetivo de favorecer la adquisición de competencias laborales y fomentar la empleabilidad; b) una mejora en las condiciones laborales y representatividad más equitativas, en todos los ámbitos de participación; c) un llamamiento a la responsabilidad social de las empresas y al impulso de mecanismos favorecedores del acceso al mercado laboral tales como fomentar los incentivos en Centros Especiales de Empleo, el Empleo con Apoyo y la Formación Ocupacional, aumentar la reserva de plazas y cuotas a empresas por la contratación, entre otros.

En relación al proceso de identificación de las acciones locales, para la inserción laboral de mujeres con diversidadfuncional, llevadas a cabo por las instituciones compostelanas de referencia, se aplica la guía especificada en el apartado anterior. Su uso nos permitió extraer información relevante sobre la calidad de los recursos que ofrecen a través de sus webs, programas y líneas de intervención, sobre la diversidad de los contenidos que trabajan, así como sobre las actuaciones que contem plan para el desarrollo de los procesos de inserción laboral del colectivo.

En términos generales identificamos que, en su mayoría, son páginas web que ofrecen la posibilidad de adaptar sus recursos y contenidos a las necesidades visuales, auditivas y/o cognitivas que puedan demandary/o presentar sus usuarias. Son portales muy intuitivos, bien organizados y distribuidos de tal forma que facilitan la navegación por los mismos, con un dominio informático bastante básico. Lo mismo sucede con los espacios físicos, donde su estructura arquitectónica, accesos, distribuciones, conectividad y usos están diseñados para facilitar el tránsito y habitabilidad de sus usuarias en función de sus necesidades particulares.

Tanto en los escenarios virtuales analizados, como en los escenarios presenciales, todos los recursos y materiales disponibles incluyen información relativa a la misión y objetivos de cada entidad. También ofrecen espacios destinados a incentivar y fomentar la participación familiar en las actividades de información-formación, así como el acceso para la consulta y navegación por otras páginas y recursos de interés. Realizan una importante visibilización del colectivo al que atienden, defendiendo e informando sobre sus derechos y promoviendo la igualdad de oportunidades. Para ello, mantienen un contacto y colaboración permanente con otras entidades, recursos humanos y servicios externos, que facilitan el empleo a través de convenios de contratación con empresas próximas, Centros Especiales de Empleo o el incentivo laboral de la modalidad de Empleo con Apoyo; obteniendo resultados positivos que se pueden consultar en la mayońa de las páginas. Y aunque solo una de ellas aborda de forma integral la inserción laboral del colectivo femenino, todas ellas incluyen la perspectiva de género en sus intervenciones y servicios. Realizan una importante labor para destacar las potencialidades que ofrece la contratación de personas con diversidad funcional, dejando constancia de que sus habilidades- reforzadas todas mediante procesos formativos certificados e impartidos en las mismas-son las mismas que las que pueda presentar cualquier otra persona en dichos puestos. Esta acción de desarrollo local funciona porque tienden puentes de empleabilidad con las empresas y entidades del entomo próximo, obteniendo información de primera mano sobre sus ofertas de empleo y sobre los requerimientos que deben cumplir las personas que estén interesadas en optar a ellas.

Para cerrar este estudio se mantienen una serie de entrevistas con los informantes clave, con el propósito de conocer qué tipo de acciones, servicios y líneas de intervención se siguen en estas institucionesy cómo se pueden mejorar. En todas las entrevistas, cuando se les pregunta sobre el porcentaje de demandantes mujeresque acuden y gestionan desde sus entidades, todas nos mencionan que hoy en día los porcentajes de participación femenina son prácticamente iguales que en el caso del colectivo masculino. No obstante, en el caso de las acciones vinculadas a la inserción laboral, hay un porcentaje mayor de hombres que las demandan frente al número de mujeres que acuden solicitando ese servicio. En estas entidades todas las intervenciones que demandan servicios, apoyos y recursos para los procesos de inserción laboral se realizan de un modo semejante, adaptándose siempre a las necesidades y particularidades de cada persona, con independencia del colectivo o género del que formen parte; a excepción de aquellas acciones específicas que síse desarrollan bajo el paraguas de la incorporación de la perspectiva de género y/o discriminación positiva.

Consideran que, aunque en principio todas las demandas se abordan de igual forma, si existen 
factores sociales, culturales y laborales determinantes que inciden de forma diferente a la hora de trabajar con la persona, dependiendo de si el demandante es un hombre o una mujer. Es decir, nos comentan que en el caso de las demandantes mujeres es frecuente que deban recurnir a un trabajo adicional con las familias, ya que en estos casos son más propensas a ejercer una sobreprotección sobre ellas frente a la que ejercen cuando los demandantes son varones. Con respecto a las actuaciones concretas que llevan a cabo con sus usuarias demandantes, para estas profesionales resulta fundamental prionizar la formación en habilidadesy competencias laborales que les permitan enfrentarse, con unas mínimas garantías de éxito, a los procesos selectivos de acceso al empleo. Inciden en que el nivel formativo con el que acceden al empleo es bastante bajo y poco cualificado, lo que representa una de sus principales dificultades de inserción laboral. Identificany analizan, de forma minuciosa, cada oferta laboral para conocer la formación que demandan, la información que debe dominar la candidata para acceder a ese puesto y las tareas, funciones y responsabilidades que conforman ese empleo para que puedan mantenerlo. Para ellas, conocer a fondo todos estos aspectos es clave porque les permiten mejorar la adecuación del perfil formativo de las mujeres al perfil profesional del trabajo, además de reforzar la autoestima de este colectivo.

También consideran relevante sensibilizar y concienciar a la sociedad y, en concreto, al ámbito empresarial, para erradicar la cultura lacerante y discriminatoria de los estereotipos y roles de género, todavía presentes en nuestras sociedaddes. Una vez más, los estereotipos y estigmas vuelven a posicionarse como esas grandes barreras que dificultan el logro de la inserción laboral, pues están presentes en el ámbito empresarial, en el familiar y en el social. Esta realidad se complica en el caso de nuestro colectivo, pues se tiende a asociar a estas mujeres con un alto grado de dependencia, unasobreprotección familiar, el absentismo laboral o el cuidado permanente de sus familiares. A estos factoresse le suman las dificultades añadidas de residir en zonas rurales, ya que al no poseer un carnet de conducir o un coche propio, dependen permanentemente del transporte urbano o de que alguien las lleve al trabajo.

En cuanto a la gestión económica de su trabajo diario, las entrevistadas nos cuentan que muchas veces sus programas y talleres formativos se apoyan en incentivos externos, tales como las subvenciones Estatales, Autonómicas, de las Diputaciones y/o entidades privadas. También que establecen acuerdos de prácticas y cursos formativos con otras empresas del entomo próximo, acordando con las mismas- en ocasiones- una contratación posterior. Para todo ello disponen de sus propios Servicios de Intermediación Laboral o utilizan los facilitados por las entidades colaboradoras, que se encargan de acompañar, ayudar y asesorar a la demandante durante todo el proceso de inserción laboral.

Finalmente, nos indican como propuestas de mejora seguir trabajando en la concienciación de la población, para que empaticen más con las problemáticas y realidad en la que viven estas mujeres. También que se deben incentivar mayores ayudas económ icas para costear por ejemplo el desplazamiento en las zonas rurales, desmitificar las reticencias familiares, abordar la conciliación en el ámbito doméstico y el laboral a través de procesos de coeducación y, en definitiva, mejorar la percepción sociolaboral que se tiene del colectivo objeto de estudio.

\section{CONCLUSIONES FINALES: UN DECÁLOGO DE BUENAS PRÁCTICAS}

Como conclusiones finales podemos destacar, en primer lugar, que las discriminaciones, estereotipos y estigmas que afectan a este colectivo en ámbitos como el familiar, el educativo y el laboral, se relacionan con creenciassocialmente establecidas que se reproducen generación tras generación. Suponen las mayores trabas para el logro de la inserción laboral y provocan que estas mujeres deban afrontar una doble discriminación: la de género y la de diversidad funcional. En segundo lugar, la imagen que el tejido empresarial suele tener sobre estas mujeres está distorsionado con respecto a la diversidad funcional; es decir, transmiten una construcción social errónea de su diversidad, empleándola como la base que justifica su exclusión laboral y/o las representaciones culturales que se tienen sobre las limitaciones laborales que se le atribuyen. Su diversidad funcional siempre les resta facultades y siempre les suma dificultades a las acciones discriminatorias que ya de por sí sufren las mujeres. Nos referimos a las bajas tasas de ocupación, a la menor estabilidad laboral y a la escasa discriminación retributiva que, entre otras desigualdades, siguen presentes en el ámbito laboral del colectivo femenino (Mariño, 2009; López, 2005; Cedrero, 2017).

La brecha género- diversidad funcional sigue presente en nuestra sociedad, porque el mercado 
laboral no favorece la inserción del colectivo de mujeres con diversidad funcional. Siguen considerando que debido al desajuste y/o falta de competencias profesionales, su valía y productividad es un factor negativo, cuando en realidad su incorporación al conjunto de trabajadores y trabajadoras de las empresas, suma valores positivos tales como compañerismo, empatía o felicidad. Las entidades y profesionales que trabajan por y para su inserción laboral, manifiestan la necesidad de comenzar a formarlas en el desarrollo de su competencia vocacional a tres niveles: habilidades para elegir una profesión, conociéndose a sí mismas y su mercado laboral más inmediato (formación); habilidades para la obtención y acceso a un puesto laboral (búsqueda de empleo) y habilidades para el mantenimiento del trabajo (hábitos laborales). (Díez, Rodríguez, Cam po y Martínez, 2017). También se hace necesario mejorar la coordinación entre las diferentes estructuras gubernamentales e instituciones para el empleo, públicas o privadas, estatales o provinciales, para que mejoren el cumplimiento del derecho laboral de toda persona a una inserción plena en igualdad de oportunidades y condiciones (Buedo, 2015).

Con toda la información recogida y analizada a lo largo de este estudio, consideramos oportuno presentar, a continuación, un compendio de retos y mejoras que se deben tener en cuenta, para la consecución de una exitosa inserción laboral del colectivo de mujeres con diversidad funcional en la comarca compostelana:

- Reforzar el acompañamiento y orientación en las estrategias de búsqueda y acceso al empleo, tales como elaboración del currículum vitae, carta de presentación, entrevista y procesos selectivos, etc. para reducir las inseguridades que manifiesta este colectivo de cara a la realización de su primera entrevista laboral.

- Incluir en las acciones formativas un ajuste hacia las competencias profesionales más demandadas; sobre todo en las ofertas laborales reales presentes en el mercado laboral más próximo, para que se mejore con eficacia su empleabilidad.

- Mejorar su formación en competencia digital y dominios tecnológicos para actualizar su formación acorde a los requerimientos de una sociedad moderna. Integrar nuevos recursos digitales a los ya existentes, como por ejemplo a las propias páginas web, o como material complementario a la formación presencial/virtual. La adquisición de competencias informáticas es un elemento curri- cular muy valorado en los procesos de inserción laboral.

- Partir de un enfoque de género en todos los servicios, recursos y procesos presentes en las acciones de inserción laboral de las mujeres con diversidad funcional. A estas mujeres les resulta muy difícil y complejo dar con una solución adecuada para superar las barreras, desigualdades y circunstancias derivadas de la doble discriminación. Por ello, dotarlas de conocimientos e información en calve de género, se convierten en herramientas muy potentes y valiosas para que, desde su puesto laboral, contribuyan a la erradicación de las discriminaciones, agravios y estereotipos todavía presentes en las actuales esferas sociales y mercados de trabajo.

- Orientar, informar y apoyar todas las iniciativas asociativas y de emprendimiento femeninas que surjan en esta comarca. El autoempleo se posiciona, así, como un prodigioso método de inserción laboral.

- Sensibilizar al personal trabajador de las empresas y entidades, hacia la comprensión del derecho a la igualdad de oportunidades de las mujeres con diversidad funcional. En este escenario, la modalidad de empleo con apoyo puede convertirse en la medida idónea para la eliminación de estereotipos en el puesto laboral.

- Reforzar la labor de visibilización del colectivo, fomentando que sean las propias mujeres con diversidad funcional las que se expresen y sean protagonistas de su proceso de cambio, implicándose de forma activa.

- Defender los derechos de todas las mujeres con diversidad funcional, impulsando la creación de políticas locales más ambiciosas y resolutivas, que de verdad amparen su situación y les proporcionen verdaderas oportunidades de inserción laboral como el derecho universal que es.

- Potenciar el trabajo en red, transversal y colaborativo entre instituciones del mismo y/o diversos ámbitos, para que, bajo objetivos comunes de inserción laboral, puedan obtener conjuntamente mejores resultados en el entomo empresarial.

Finalmente creemos que se hace necesario realizar una mayor inversión en recursos y espacios para la investigación y estudio de esta temática y sus problemáticas, conociendo la realidad sociolaboral de cada entorno y utilizando la 
información obtenida para ayudar a la mejora de las intervenciones que desarrollan las entidades.

\section{NOTAS}

${ }^{1}$ Se parte de la guía para el análisis de materiales didácticos digitales del "Proyecto Escuel@ Digit@l" (consultado en el artículo de Cepeda, Gallardo y Rodríguez,2017), se mantiene su estructura original, pero se reelaboran los contenidos de los ítems que la configuran al objeto de estudio de este trabajo.

\section{REFERENCIAS BIBLIOGRÁFICAS}

- ACADAR (2018). ACADAR. http://www.acadar.org/

- Buedo Martínez, S. (2015). Mujeres y mercado laboral en la actualidad, un análisis desde la perspectiva de género: genéricamente empobrecidas, patriarcalmente desiguales. Revista de Educación Social, (21), 64-86.

https://eduso.net/res/wpcontent/uploads/documentos/680.pdf

- Carrizosa, J, y Sesé, A. (2014). La diversidad funcional en el trabajo. Educación Social. Revista de intervención socioeducativa, (58), 65-79. Recuperado de: https://www.raco.cat/index.php/Educacio Social/a rticle/view/284936/373093.

- Cedrero Uceda, L. A. (2017). La discapacidad como factor de discriminación en el ámbito laboral. [Tese de Doutoramento, Universidade Complutense de Madrid]. Dialnet.

https://dialnet.unirioja.es/servlet/tesis? codigo $=1$ 27449

- Cepeda Romero, O., Gallardo Fernández, I. M., y Rodríguez Rodríguez, J. (2017). La evaluación de los materiales didácticos digitales. RELATEC, 16(2), 79- 95. DOI: https://doi.org/10.17398/1695-288X.16.2.79.

- CERMI (2005). I Plan Integral de Acción de Mujeres con Discapacidad 2005-2008. CERMI. https://www.cermi.es/sites/default/files/docs/col ecciones/Libro188905.pdf.

- CERMI (2013). II Plan Integral de Acción de mujeres con discapacidad 2013-2016. CERMI. http://www.fundacioncermimujeres.es/sites /d efa ult/files/ii_plan_integral_de_accin_de_mujeres_defi nitivo.pdf.

- Confederación Galega de Personas con Discapacidad (2017). COGAMI.

http://www.cogami.gal/.

- Instituto Nacional de Estadística (2018). El Empleo de las Personas con Discapacidad (EPD).

https://www.ine.es/prensa/epd_2018.pdf

- Díez, E., Rodríguez, N., Campo, M e Martínez, I. (2017). Formación online para jóvenes con discapacidad sobre competencias transversales y empleabilidad bajo los principios del diseño universal para el aprendizaje. En Rodríguez-Martín, A (Coord.). Prácticas innovadoras inclusivas: retos y oportunidades (pp.2671-2676). Universidad de Oviedo.

http://digibuo.uniovi.es/dspace/bitstream/10651 /50342/4/FormacionOnline.pdf.

- FADEMGA- Plena Inclusión Galicia (s.f). FADEMGA. https://www.fademga.org/gl/.

- Fundación Down Compostela (s.f). DOWN Compostela. https://www.downcompostela.org/.

- Mariño Fernández, R. (2009). Análisis de la trayectoria formativa de la mujer en las ramas industriales de FP en Galicia y su inserción sociolaboral [Tese de Doutoramento, Universidade de Santiago de Compostela]. Repositorio Minerva. https://minerva.usc.es/xmlui/bitstream/handle/1 0347/2603/9788498873030_cont ent.pdf? sequence $=1 \&$ is Allowed $=\mathrm{y}$.

- López, M. (2005). Discapacidad y empleo en España: su visibilidad. Revista Innovar de Ciencias Administrativas y Sociales, 15(26), 59-72. DOI: http://dx.doi.org/10.1400/222458.

- Observatorio Estatal de la Discapacidad (2018). Informe Olivenza 2019, sobre la situación general de la discapacidad en España.

https://www.observatoriodeladiscapacidad.info/i nforme-olivenza-2019-obre-la- situacion-generalde-la-discapacidad-en-espana/.

- ONU (2015). Objetivos de desarrollo sostenible de la organización de las naciones unidas. https://www.un.org/sustainabledevelopment/es/.

- PNUD (2016). Informe sobre desarrollo humano. https://www.undp.org/content/undp/es /home/li brarypage/hdr/2016-human- developmentreport.html.

- Portillo, I., Shum, G., Conde, Á., y Lobato, H. (2006). Mujer, discapacidad y empleo: tejiendo la discriminación. Acciones e investigaciones sociales, 22(extra-1), 187-188.

https://dialnet.unirioja.es/servlet/articulo?codigo $=2125759$.

- Romañach, J, y Palacios, A. (2008). El modelo de la diversidad: una nueva visión de la bioética desde la perspectiva de las personas con 
diversidad funcional (discapacidad). Intersticios. Revista sociológica de pensamiento crítico, 2(2). http://www.intersticios.es/article/view/2712

- Serra, L.M. (2014). Feminismo y discapacidad. Derechos y libertades: revista de filosofía del derecho y derechos humanos, (31), 251-272. DOI: http://dx.doi.org/10.1400/222458.

- Soler, A, Teixeira, T, y Jaime, V. (2008, 27-29 de marzo). Discapacidad y dependencia: una perspectiva de género [ponencia]. XI Jornadas de Economía Crítica, Bilbao.

http://www.repositoriocdpd.net:8080/handle/12 $3456789 / 746$. 